REVISTA MATEMÁTICA de la

Universidad Complutense de Madrid

Volumen 8, número 2: 1995

http://dx.doi.org/10.5209/rev_REMA.1995.v8.n2.17657

\title{
Aleatoreidad e Inmunidad
}

\author{
J.F. PRIDA
}

\begin{abstract}
By introducing the concept of randomness through notions of recursion theory, the set of the random numbers is effectively inmune. The proof of this well-known result makes an essential use of the recursion theorem. In this paper, randomness is introduced starting from the more common notion of definability in Robinson's arithmetic and the same result is obtained using an extension of the fixed-point theorem, which we prove at the end of the paper. Finally we define a recursive function dominating the set of the random numbers, which consequently is not hyperinmune.
\end{abstract}

Sea $L$ el lenguaje usual de la aritmética con $\left\{x_{i}: i \in \omega\right\}$ como conjunto de variables, donde los símbolos " $o$ " y " $s$ " denotan el número cero y la función sucesor. Los números naturales serán denotados mediante los términos, " $o$ ", "so", "sso", etc... y n será el nombre del término que denota el número n. Sea $\left\{\alpha_{i}\left(x_{o}\right): i \in \omega\right\}$ el conjunto de fórmulas de $L$ cuya única variable libre es $x_{o}$ y sea finalmente $Q \subset L$ la aritmética de Robinson.

1991 Mathematics Subject Classification: 03D20

Servicio publicaciones Univ. Complutense. Madrid, 1995. 
Definición 1. La fórmula $\alpha_{k}\left(x_{o}\right)$ define en $Q$ el número natural $j$ si $\forall x_{o}\left(\alpha_{k}\left(x_{o}\right) \leftrightarrow x_{o} \equiv \mathbf{j}\right)$ ” es un teorema de $Q$.

Definición 2. El número natural $j$ es aleatorio si para todo $k \in \omega$ se verifica: si $\alpha_{k}\left(x_{o}\right)$ define $j$, entonces $k$ es igual o mayor que $j$. El conjunto de los números aleatorios será denotado por $R$.

Sea $\left\{\phi_{i}: i \in \omega\right\}$ el conjunto de las funciones recursivas parciales de $\omega$ en $\omega$ y sea $W_{i}$ el dominio de $\phi_{i}$.

Definición 3. Un conjunto $A$ es efectivamente inmune si es infinito y existe una función recursiva $f$ tal que para todo $x \in \omega$ verifica:

$$
W_{x} \subset A \rightarrow\left|W_{x}\right| \leq f(x)
$$

Definición 4. Un conjunto es hiperinmune si es infinito y no está dominado por ninguna función recursiva.

Teorema. El conjunto $R$ de los números aleatorios es efectivamente inmune ${ }^{1}$, pero no hiperinmune.

En primer lugar probaremos que $R$ es infinito.

Definición $5 . \quad \delta: \omega \rightarrow \omega$ es la función (recursiva parcial) formada por los pares $(j, k)$ tales que $\alpha_{j}\left(x_{0}\right)$ define $k$ en $Q$.

Definición 6. $C_{a}$ es el conjunto $\left\{b \in \omega:(\exists n \in \omega): \delta^{n}(b)=a\right\}^{2}$.

Definición 7. Para todo $a \in \omega \quad a^{\star}$ es el menor elemento de $C_{a}$.

De $a^{\star} \in C_{a}$ y $a^{\star \star} \in C_{a^{\star}}$ se sigue que $a^{\star \star} \in C_{a}$, con lo que $a^{\star} \leq a^{\star \star}$ $\mathrm{y}$ en consecuencia

$$
a^{\star}=a^{\star \star}
$$

Definición 8. S e s el conjunto $\left\{a^{\star}: a \in \omega\right\}$.

${ }^{1}$ Este resultado es conocido en el caso en que se ha definido $R$ como el conjunto $\left\{x: \forall y\left(\phi_{y}^{0}=x \rightarrow y \geq x\right)\right\}$ (cfr. [1], p. 265).

${ }^{2} \mathrm{Si}$ para todo $A \subset \omega$ definimos $C A$ como el conjunto $\{b \in \omega:(\exists n \in$ w) $\left.\delta^{n}(b) \in A\right\}$, entonces $C$. define una topología en $2^{\omega}$, al verificarse: $A \subset C A, C C A=C A, C \emptyset=\emptyset$ y $C(A \cup B)=C A \cup C B$. 
De (2) se sigue inmediatamente:

$$
S=\left\{a: a=a^{\star}\right\} .
$$

Claramente $S \subset R$. Además $S$ es un conjunto $\Pi_{1}$, ya que $S=\left\{a: \forall b \forall n\left(\delta^{n}(b)=a \rightarrow b \geq a\right)\right\}$.

Definición 9. Dos números $a, b$ son independientes si verifican:

$$
a \notin C_{b} \quad \text { y } \quad b \notin C_{a} .
$$

Si $a$ y $b$ son independientes, entonces:

$$
\begin{gathered}
C_{a} \cap C_{b}=\emptyset \\
a^{\star} \neq b^{\star} .
\end{gathered}
$$

En efecto:

$$
\text { (4) } \Rightarrow(5)
$$

$e \in C_{a} \cap C_{b} \Rightarrow$ existen $m, n: \delta^{m}(e)=a$ y $\delta^{n}(e)=b$, con lo que $m \geq n$ implica $b \in C_{a}$ y $n \geq m$ implica $a \in C_{b}$.

(5) $\Rightarrow(6)$

Como $a^{\star} \in C_{a}$ y $b^{\star} \in C_{b}, \quad a^{\star}=b^{\star}$ implica $C_{a} \cap C_{b} \neq \emptyset$.

Sea $h$ la función inyectiva y recursiva tal que $\alpha_{h(j)}(x o)$ es la fórmula " $\forall x_{j}\left(x_{o} \equiv x_{j}\right)$ ". Puesto que para ningún $n \in \omega$ la fórmula

" $\forall x_{0}\left(\forall x_{j}\left(x_{0} \equiv x_{j}\right) \leftrightarrow x_{0}=\mathrm{n}\right)$ " es un teorema de $Q, \delta h(j)$ está indefinida para todo $j \mathrm{y}$, en consecuencia, $i \neq j$ implica que $h(i) \notin C_{h(j)}$ y $h(j) \notin C_{h(i)}$, con lo que, al verificar (4), $h(i)$ y $h(j)$ son independientes $y$, por tanto,

$$
h(i)^{\star} \neq h(j)^{\star} .
$$

Definición 10. H es el conjunto $\left\{h(j)^{\star}: j \in \omega\right\}$. 
$H$ es, pues, un subconjunto de $S$ (y por tanto de $R$ ), que además, de acuerdo con (7), es infinito.

En segundo lugar probaremos que $H$ (y por tanto $S$ y $R$ ), está dominado por la función recursiva $g$ definida por la identidad

$$
g(n)=h(0)+h(1)+\ldots+h(n) .
$$

En efecto, si $H=\left\{a_{o}<a_{1}<a_{2}<\ldots\right\}$, se tendrá:

$$
\begin{aligned}
g(n) & =h(0)+h(1)+\ldots+h(n) \\
& \geq h(0)^{\star}+h(1)^{\star}+h(n)^{\star} \\
& \geq a_{0}+a_{1}+\ldots+a_{n} \geq a_{n}{ }^{3}
\end{aligned}
$$

Finalmente demostraremos la existencia de una función recursiva que controla los cardinales de los conjuntos recursivamente enumerables contenidos en $R$, i.e, que verifica (1).

Con tal fin definimos $\phi: \omega^{2} \rightarrow \omega$ a través del siguiente algoritmo: Dado el par $(e, n) \in \omega^{2}$, mediante computación simultanea de $\phi_{e}(0)$, $\phi_{e}(1), \phi_{e}(2), \ldots$ se van listando elementos de $W_{e}$. Si $\left|W_{e}\right| \leq n+1$, la computación termina cuando hayan aparecido $n+2$ elementos distintos, en cuyo caso el valor asignado a $\phi(e, n)$ será el mayor de ellos. Si $|W e| \leq$ $n+1$, la computación no tiene fin. Así pues, si $\left|W_{e}\right|>n+1$ se verifica:

$$
\begin{gathered}
\phi(e, n) \downarrow \\
\phi(e, n) \in W_{e} \\
\phi(e, n)>n .
\end{gathered}
$$

Sea $\beta\left(x_{0}, x_{1}, x_{2}\right)$ una fórmula de $L$ tal que si $\phi(e, n)=m$, entonces $\beta\left(x_{o}, \mathbf{e}, \mathbf{n} \leftrightarrow x_{o} \equiv \mathbf{m}\right)$ es un teorema de $Q$. De acuerdo con el teorema

\footnotetext{
${ }^{3}$ Idéntico razonamiento puede utilizarse para probar que $R$ no es hiperinmune cuando ha sido definido como en la nota (2). En tal caso $h$ es una función recursiva tal que $\phi_{h(j)}^{0}$ está indefinida para todo $j$.
} 
de recursión de la aritmética (que demostramos a continuación), existirá una función recursiva $r: \omega \rightarrow \omega$ tal que para todo $a \in \omega$ verifica:

$$
\vdash^{Q} \alpha_{r(a)}\left(x_{0}\right) \leftrightarrow \beta\left(x_{0}, \mathbf{a}, \mathbf{r}(\mathbf{a})\right)
$$

Bajo la hipótesis de que $\left|W_{e}\right|>r(e)+1$. de (8), (9), y (10) se obtiene:

$$
\begin{gathered}
\phi(e, r(e)) \downarrow \\
\phi(e, r(e)) \in W_{e} \\
\phi(e, r(e))>r(e) \\
\vdash^{Q} \beta\left(x_{o}, \mathbf{e}, \mathbf{r}(\mathbf{e})\right) \leftrightarrow\left(x_{o} \equiv \phi(\mathbf{e}, \mathbf{r}(\mathbf{e}))\right) .
\end{gathered}
$$

De (11) y (15) se sigue:

$$
\vdash^{Q} \alpha_{r(e)}\left(x_{o}\right) \leftrightarrow\left(x_{o} \equiv \phi(\mathbf{e}, \mathbf{r}(\mathbf{e}))\right)
$$

con lo que $\alpha_{r(e)}\left(x_{0}\right)$ define $\phi(e, r(e)) \mathrm{y}$, por tanto, de acuerdo con (13), (14) y la definición (2),

$$
\phi(e, r(e)) \in \bar{R} \cap W_{e}
$$

lo que implica que $W_{e}$ no está contenido en $R$. Así pues

$$
W_{e} \subset R \quad \Rightarrow \quad\left|W_{e}\right| \leq r(e)+1
$$

En consecuencia $H, S$ y $R$ son efectivamente inmunes (y los complementarios de $S$ y $R$ efectivamente simples), pero no hiperinmunes. 
Teorema de recursión de la aritmética. Si $L$ es el lenguaje de la aritmética $y\left\{\alpha_{i}(x): i \in \omega\right\}$ es una enumeración efectiva de las fórmulas de $L$ con cuya única variable libre es $x$, para toda fórmula de $L$ con $n+2$ variables libres $\beta\left(x, y_{1}, \ldots, y_{n}, z\right)$ existe una función recursiva $r: \omega^{n} \rightarrow \omega$ tal que para todo $a_{1}, a_{2}, \ldots, a_{n}$ se verifica:

$$
\vdash^{Q} \alpha_{r\left(a_{1}, a_{2}, \ldots, a_{n}\right)}(x) \leftrightarrow \beta\left(x, \mathbf{a}_{1}, \mathbf{a}_{2}, \ldots, \mathbf{a}_{\mathbf{n}}, \mathbf{r}\left(\mathbf{a}_{1}, \mathbf{a}_{2}, \ldots, \mathbf{a}_{\mathbf{n}}\right)\right) .
$$

\section{Demostración:}

Sean $a$ e $y$ abreviaturas de $a_{1}, a_{2}, . ., a_{n}$ e $y_{1}, y_{2}, . ., y_{n}$ y sea $\left\{\gamma_{i}(x, y, z)\right.$ : $i \in \omega\}$ una enumeración efectiva de las fórmulas de $L$ cuyas variables libres son $x, y, z$.

Definición 11. $g: \omega^{n+1} \rightarrow \omega$ es la función recursiva tal que $\alpha_{g(a, b)}(x)$ es la fórmula $\gamma_{b}(x, \mathbf{a}, \mathbf{b})$, lo que se expresará de la forma

$$
g(a, b)=\left\lceil\gamma_{b}(x, \mathbf{a}, \mathbf{b})\right\rceil
$$

Definición 12. $\delta(x, y, z)$ es la fórmula que representa a $g$ en $Q$, i.e., tal que

$$
g(m, n)=s \quad \Rightarrow \quad \vdash^{Q} \forall x(\delta(x, \mathbf{m}, \mathbf{n}) \leftrightarrow x \equiv \mathbf{s}) .
$$

Sea $q$ el número natural tal que $\gamma_{q}(u, y, z)$ es la fórmula

$$
\forall u(\delta(u, y, z) \rightarrow \beta(x, y, u))
$$

y sea $r: \omega^{n} \rightarrow \omega$ la función recursiva tal que para todo $a \in \omega$

$$
r(a)=\left\{\gamma_{q}(x, \mathbf{a}, \mathbf{q})\right\rceil=\lceil\forall u(\delta(u, \mathbf{a}, \mathbf{q}) \rightarrow \beta(x, \mathbf{a}, u)\rceil
$$

Se tiene sucesivamente:

[0] $g(a, q)=\left\lceil\gamma_{q}(\boldsymbol{x}, \mathbf{a}, \mathbf{q})\right\rceil=r(a)$ 
[1] $\quad \vdash^{Q} \forall x(\delta(x, \mathbf{a}, \mathbf{q}) \leftrightarrow x \equiv \mathbf{r}(\mathbf{a}))$

[2] $\quad \quad^{Q} \delta(\mathbf{r}(\mathbf{a}), \mathbf{a}, \mathbf{q})$

[3] $\quad \vdash^{Q} \alpha_{r(\mathbf{a})}(x) \rightarrow(\delta(\mathbf{r}(\mathbf{a}), \mathbf{a}, \mathbf{q}) \rightarrow \beta(x, \mathbf{a}, \mathbf{r}(\mathbf{a})))$

[4] $\quad \vdash^{Q} \alpha_{\mathbf{r ( a )}}(x) \rightarrow \beta(x, \mathbf{a}, \mathbf{r}(\mathbf{a}))$

[5] $\quad \vdash^{Q} \beta(x, \mathbf{a}, \mathbf{r}(\mathbf{a})) \rightarrow(u \equiv \mathbf{r}(\mathbf{a}) \rightarrow \beta(x, \mathbf{a}, u)) \quad$ (tautología)

[6] $\quad \vdash^{Q} \delta(u, \mathbf{a}, \mathbf{q}) \leftrightarrow u \equiv \mathbf{r}(\mathbf{a})$

[7] $\quad \vdash^{Q} \beta(x, \mathbf{a}, \mathbf{r}(\mathbf{a})) \rightarrow(\delta(u, \mathbf{a}, \mathbf{q}) \rightarrow \beta(x, \mathbf{a}, u))$

[8] $\quad \vdash^{Q} \beta(x, \mathbf{a}, \mathbf{r}(\mathbf{a})) \rightarrow \forall u(\delta(u, \mathbf{a}, \mathbf{q}) \rightarrow \beta(x, \mathbf{a}, u))$ (pues u no está libre en $\beta(x, \mathbf{a}, \mathbf{r}(\mathbf{a}))$ )

[9] $\quad \vdash^{Q} \beta(x, \mathbf{a}, \mathbf{r}(\mathbf{a})) \rightarrow \alpha_{r(a)}(x)$

De [4] y [0] se sigue el teorema.

\section{Bibliografía}

[1] P. Odifreddi, Classical recursion theory, North-Holland, Amsterdam 1989.

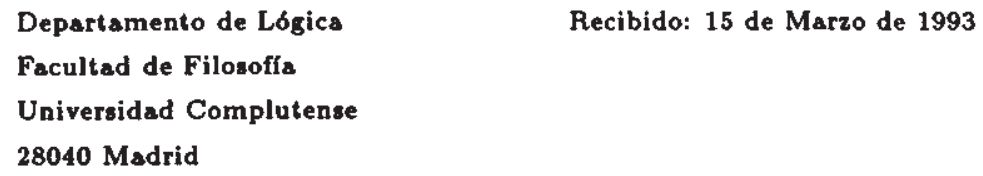

